\title{
Near infrared spectroscopy as a tool for estimation of mechanical stresses in wood
}

\author{
Jakub Sandak ${ }^{1, a}$, Anna Sandak, , , Dusan Pauliny ${ }^{1, ~ c}$, Victoria Krasnoshlyk ${ }^{2, d}$, \\ Olle Hagman ${ }^{2, ~ e}$
}

\author{
${ }^{1}$ IVALSA/CNR, via Biasi 75, 38010 San Michele All'Adige (TN), Italy \\ ${ }^{2}$ Department of Engineering Sciences and Mathematics, Lulea University of Technology, Sweden \\ a sandak@ivalsa.cnr.it, banna.sandak@ivalsa.cnr.it, 'pauliny@ivalsa.cnr.it, \\ dvictoria.krasnoshlyk@ltu.se, eolle.hagman@Itu.se
}

Keywords: mechanical stresses, wood, tension, FT-NIR, chemometry

\begin{abstract}
Significant scientific work has been dedicated for exploration of the infrared applications within wood science and technology, not much has been done linking it to the mechanical testing. It is expected that due to mechanical stresses (and related deformations) the interaction between constitutive elements of wood changes, proportionally to the stresses applied. The response of the material to mechanical stresses (such as tensile loading) on the molecular level should be therefore detectable by means of infrared spectroscopy. Dedicated tests have been devoted for proving this hypothesis. Self developed testing machine has been integrated with infrared spectrometer in order to conduct a series fully controlled mechanical tests. It was possible to predict stress level of wood during tension by applying proper chemometric analysis (partial least square (PLS) models). Implementation of infrared spectroscopy in to timber engineering and mechanical testing of wood provides very essential supplement to the typical information collected during standard tests. More additional tests and reference data is necessary in order to create more reliable and universal model suitable for routine assessments. Nevertheless, it was demonstrated that prediction of the stress on the basis of the infrared spectra is possible.
\end{abstract}

\section{Introduction}

Wood is a very complex, heterogeneous and anisotropic material. Due to the fibrous nature of the wood tissue, the way of breaking of the members is influenced by the kind of stress applied. Compression, bending and tension are typical responses of wooden members exposed into mechanical stresses. The most common causes of failure of the timber structural systems are inadequacy of configuration in relation to the actions, static and dynamic loading, besides slenderness, instability, defects of the wood, severe biotic damages, and accidental factors [1]. Inspection and evaluation of wooden structures is therefore crucial for determination of the properties of structural members and assure safety of wooden buildings. Several traditional protocols for assessment of the existing timber structures are in use. However, a strong demand for new methodologies (based on scientific and technical developments) is also recently noticed.

Proper conservation activities allow avoiding structural failures by replacement or reparation of degraded or deficient members. Conservation is multi-disciplinary effort including also recognition the importance and the vulnerability of timber structures in the preliminary phase [2]. A complete set of information regarding wooden member properties and state allows selection of the most suitable conservation procedure. Various analytical methods are employed for determination of physical properties, chemical composition or microscopic structure in conservation laboratories. However, most of the state-of-the-art techniques require sampling of objects, and therefore these are (more or less) destructive. The desired practices are focused on structure reparation and/or strengthening with minimum (or not at all) disturbance of the ancient construction. Therefore, nondestructive testing (NDT) methods find a particular place among analytical methods used today for wooden structure assessment [3]. 
Recent developments in the field of near infrared spectroscopy allow broader access of this technique in to new fields and applications. Even if significant scientific work has been dedicated for exploration of the infrared spectroscopy within wood science and technology, not much has been done linking it to the mechanical testing of wood [4]. The response to mechanical stresses on wood and cellulose fibers at the micro level has been measured nondestructively using other spectroscopic techniques. Gierlinger [5] measured Raman spectra during tensile straining of mechanically isolated fibers of spruce. She confirmed potential of such technique for monitoring changes of molecular structures while the loading was applied. Sandak et al. [6] estimated mechanical stresses on the base of mid infrared spectra and was able to predict the stress level with dedicated chemometric models.

It is expected that due to mechanical stresses (and related deformations) the interaction between constitutive elements of wood changes, proportionally to the stresses applied. The response of the material to mechanical stresses (such as tensile loading) on the molecular level should be therefore also detectable by means of FT-NIR. The goal of this work was therefore to verify if near infrared spectroscopy is suitable for detection of such inter-molecular interactions in wood polymers and to develop chemometric models for prediction of the stress levels on the base of spectra.

\section{Materials and methods}

Self developed mechanical testing machine has been developed in order to allow installation of the near infrared sensor and to conduct a series of fully controlled mechanical tests (Fig. 1). Fourier transform near infrared spectrometer Vector $22 \mathrm{~N}$ produced by Bruker Optics GmbH equipped with fiber optic probe (Fig. 1c) has been employed for scrutinizing the spectra from the surface of wood sample exposed to varying tension stresses. The spectral range measured was between $4000 \mathrm{~cm}^{-1}$ and $12000 \mathrm{~cm}^{-1}$. The spectral resolution of the spectrophotometer was $8 \mathrm{~cm}^{-1}$, each spectrum was computed as an average of 32 successive measurements.

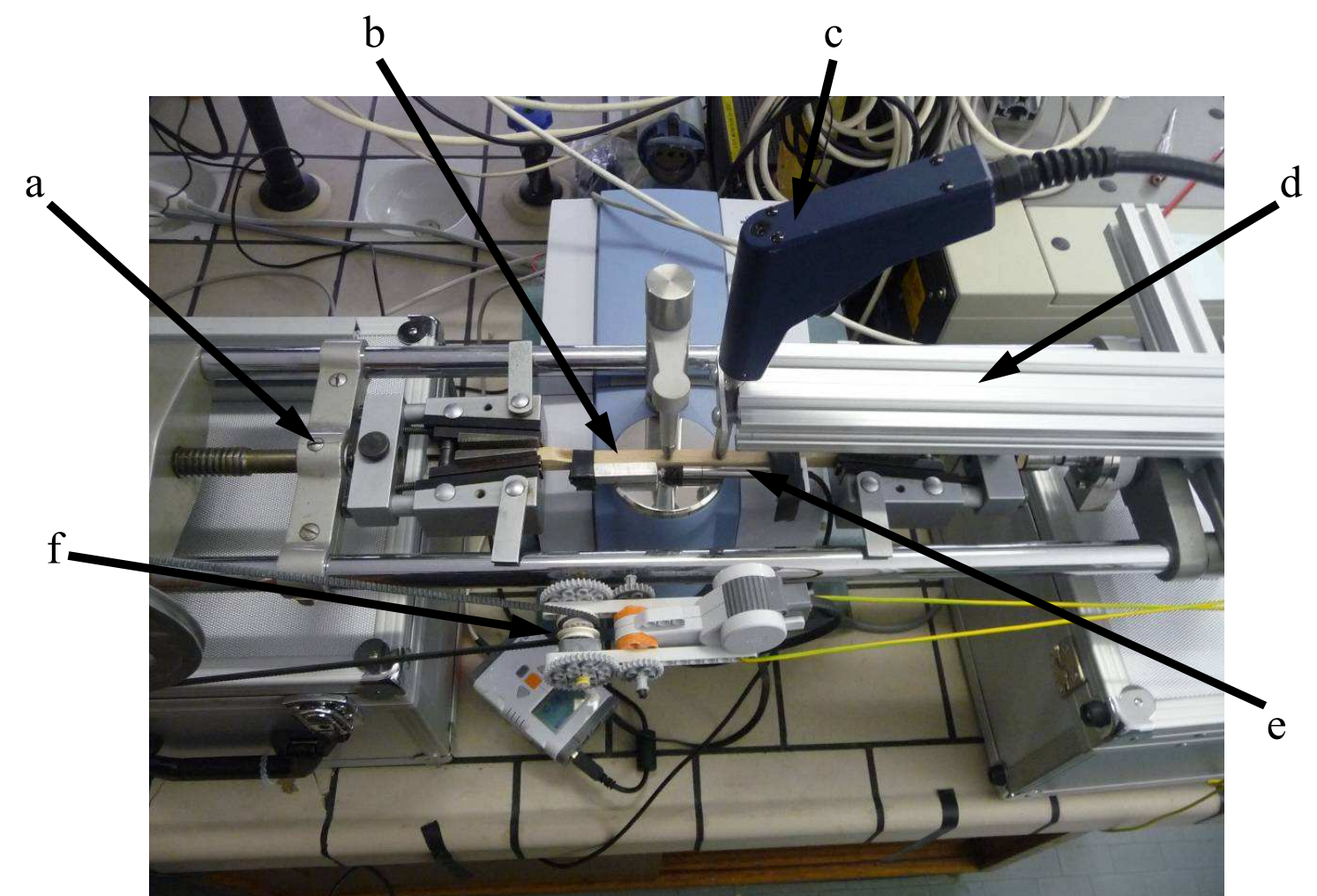

Fig. 1. Experimental set-up; mechanical testing machine (a), wooden sample (b), NIR probe (c), NIR probe holder (d), LVDT probe (e), mechanical servo (f). 
Four Norway spruce (Picea abies L. Karst) wooden blocks, dimension of the measured zone $6.5 \mathrm{~mm} \times 10 \mathrm{~mm} \times 250 \mathrm{~mm}$ (width $\times$ thickness $\times$ length respectively), have been used as experimental samples. The wood was conditioned to equilibrium moisture content of $\sim 12 \%$ in climatic chamber (room temperature $20^{\circ} \mathrm{C}$ ). All the surfaces to be measured by spectrometer have been refreshed before testing assuring a clean and smooth surface. The sample has been installed in the testing machine in the way to expose its radial plane for spectra measurement.

The sample elongation was measured in the center part of the sample (reference length $=$ $\sim 105 \mathrm{~mm}$ ) by means of LVDT (Linear Variable Differential Transformer) sensor (Fig. 1e) in order to estimate the sample deformations/strain.

Each wooden sample was tested in 5 following tension cycles. The force (stress) was gradually increasing and arrived up to $\sim 1500 \mathrm{~N}$ to assure the stress levels within elastic range. The step of increase/decrease was randomly varying between 100 to $200 \mathrm{~N}$ in each loading cycle to assure sufficient number of random reference points for developing reliable models. The force, elongation, and corresponding near infrared spectra were scrutinized and recorded for further post-processing.

Opus 6.5, LabView and SIMCA software packages were used for spectra pre-processing and data mining. 2D spectral correlation was additionally applied in order to identify all the spectra regions affected by tension forces. Finally, stress prediction models based on the Partial Least Squares algorithm were also developed.

\section{Results and discussion}

Mechanical properties of timber are highly related to its chemical composition. Simplifying, wood is a complex structure composed of three main polymers; cellulose, lignin and hemicelluloses interacting each other. Cellulose (in a form of crystalline microfibrils or amorphous regions) is generally oriented along the fiber direction and provides strength of the wood (especially in tension). Various hydrogen bonds are present in the cellulose structures what in consequence highly influences moisture affinity, hydroxyl reactivity, crystallinity of cellulose among others. The hydrogen bonds affect in consequence several physical/mechanical properties of wood on the macro scale. It is clear therefore that any deformation of cellulose chains and/or reorientation of cellulose crystals under mechanical stresses might be reviled as variation of the $\mathrm{OH}$ vibrations and to be recorded in the NIR spectra.

Lignin is cross-linking, complex aromatic polymer. As the most hydrophobic component of the cell wall, it blocks the access of water to the carbohydrates. In general lignin is stiffening wooden structures, especially in compression. Hemicelluloses are considered as chemical components linking together both, hydrophilic cellulose and hydrophobic lignin. The structure of hemicelluloses forms in wood a matrix-like structure. The contribution of hemicelluloses on the overall mechanical properties of wood is rather not significant due to its limited content, but on the other hand the hemicelluloses might be affected by deformations of cellulose chains and lignin zones as resulted to mechanical stresses. It is generally assumed that cellulose plays major role in providing strength and stiffness to wood in longitudinal tension. However, contribution of lignin and hemicellulose is more noticeable when wood is exposed to more complex (than longitudinal tension) loading modes [7].

As mentioned above, the near infrared spectra were measured on the wood samples exposed to varying mechanical stresses. The assessment of changes within these spectra might therefore provide some light in to better understanding the molecular interactions between wood polymers while loading. The simplest way to identify spectral bands most affected by mechanical loading is to regress the spectra amplitude toward the applied stresses, and to compute the resulting determination coefficients $\left(r^{2}\right)$. An example of such approach is presented in Fig. 2, where dark fields indicate spectra preprocessing/wavenumber pairs most associated to the stress level (the highest $r^{2}$ of regression). In order to define the most efficient spectra pre-processing the $r^{2}$ was computed subsequently for no-treated, normalized, first and second derivatives with 5, 15 and 25 smoothing points. The areas of dark grey color are indicating the spectral ranges the most correlated 
to mechanical stresses (darkness is proportional to value of $r^{2}$ ). By providing additional interpretation to the wavenumbers it is even possible to identify functional groups most responding to the stresses. Assessment of the band was interpreted according to Schwanninger [8].

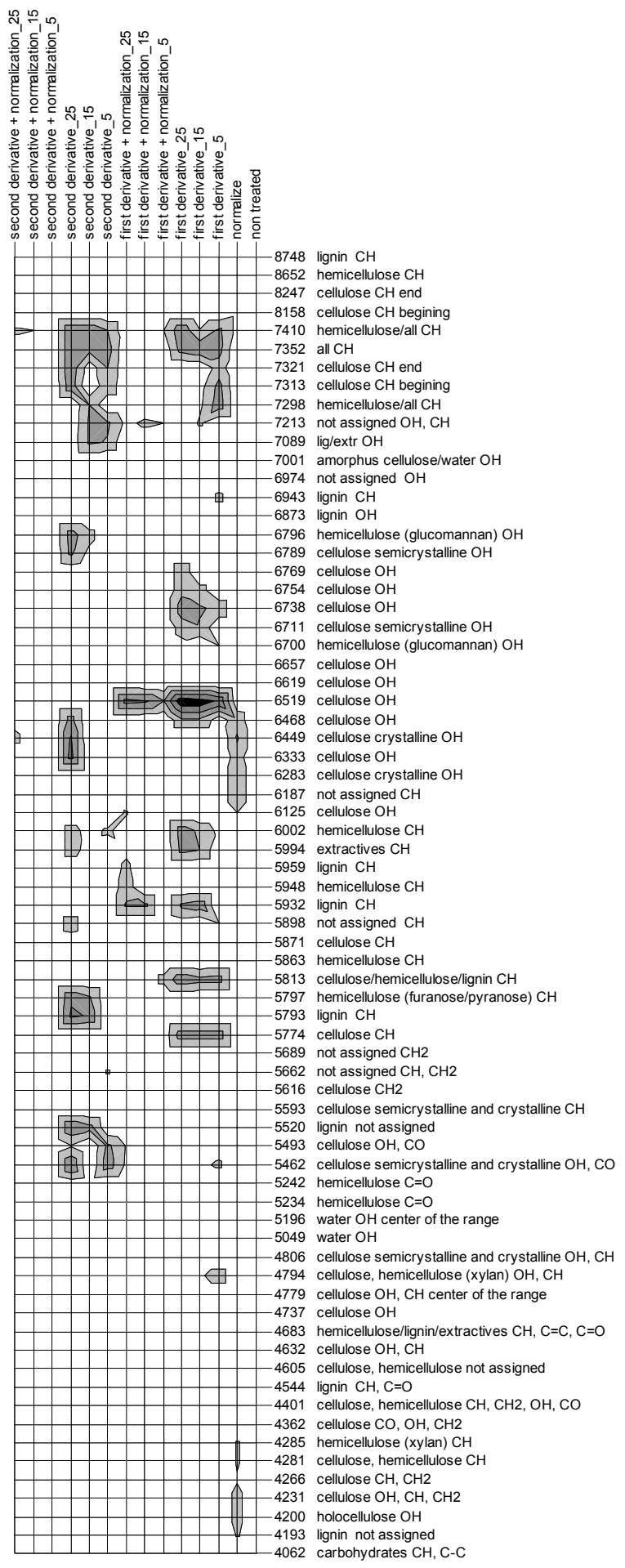

Fig. 2. Determination coefficients (r2) between NIR spectra amplitude and tension stresses in relation to the spectra pre-processing method.

The highest values of $r^{2}$ were obtained by computing first and second derivative of the spectra. The most significant changes in peak intensity (and in peak shifts) during tensile loading applied were observed after first derivative pre-processing for cellulose bands: $5776 \mathrm{~cm}^{-1}, 6520 \mathrm{~cm}^{-1}$ (the 
global highest $\left.r^{2}\right), 6472 \mathrm{~cm}^{-1}, 6740 \mathrm{~cm}^{-1}$ and range $7315-7321 \mathrm{~cm}^{-1}$. Bands corresponding to the $\mathrm{CH}$ vibration in hemicelluloses $\left(6003 \mathrm{~cm}^{-1}, 7300 \mathrm{~cm}^{-1}\right)$ were also highlighted. Additionally, peak $5816 \mathrm{~cm}^{-1}$ and $7353 \mathrm{~cm}^{-1}$ assigned to $\mathrm{CH}$ vibration in all wood components were noticed.

In case of the second derivative the highest $r^{2}$ calculated show up slightly different behavior. The most significant were regions corresponding to $\mathrm{OH}$ vibration of cellulose $\left(5464 \mathrm{~cm}^{-1}, 6334 \mathrm{~cm}^{-1}\right.$, $\left.6450 \mathrm{~cm}^{-1}, 6790 \mathrm{~cm}^{-1}\right)$, glucomannan $\left(6800 \mathrm{~cm}^{-1}\right)$, lignin/extractives $\left(7092 \mathrm{~cm}^{-1}\right)$. Some of bands related to the $\mathrm{CH}$ were also significant $\left(5795 \mathrm{~cm}^{-1}-\right.$ lignin, range $7315-7321 \mathrm{~cm}^{-1}-$ cellulose $)$ and $7353 \mathrm{~cm}^{-1}$ and $7410 \mathrm{~cm}^{-1}$ assigned to all components. The above observations were confirmed in 2D spectral correlation analysis.

The crucial role for resisting tensile stresses seems to be related to cellulose [5]. Slight shift in bands related to functional groups of cellulose was observed with increase of the stress applied. It is probably caused by the deformation of covalent bonding within the pyranose rings and other functional groups and reorientation of cellulose crystals.

The quality of stress prediction models developed on the base of the near infrared spectra was also investigated. Several chemometric methods might be applied for this purpose, but the one used in this project based on partial least squares (PLS). Clear prediction ability was observed; even if some adjustments in the models were necessary when analyze different samples (Fig. 3). Implementation of PLS models in to timber members assessment provides very essential supplement to information collected during standard tests.

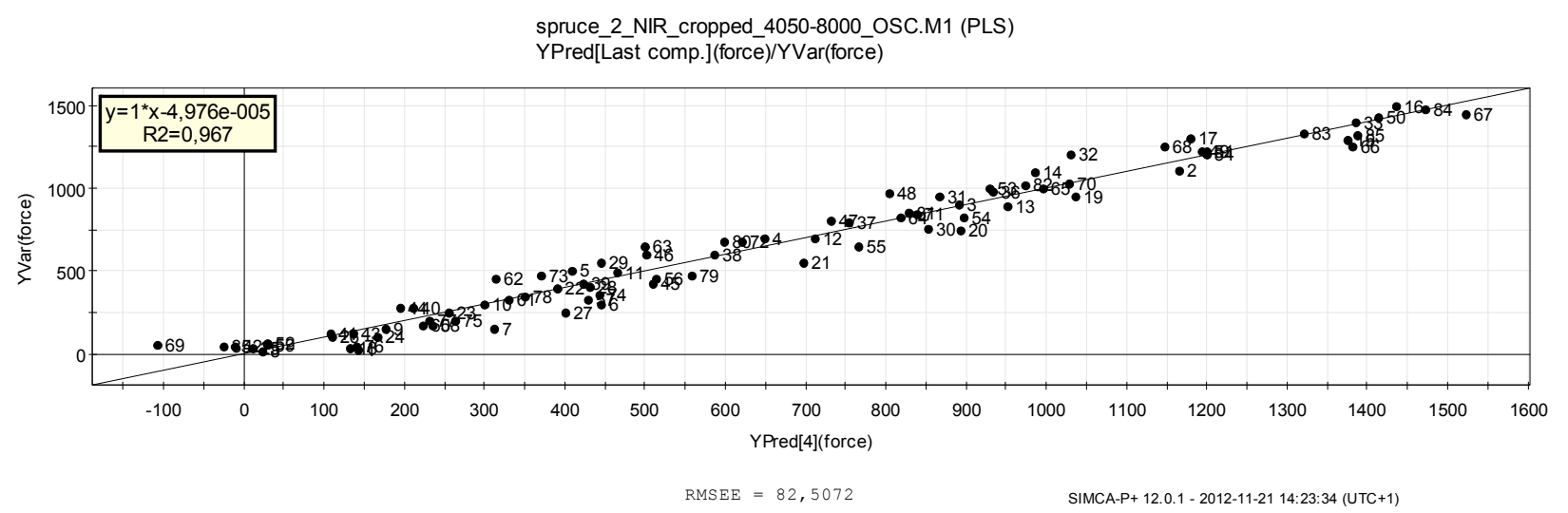

Fig. 3. Predicted vs. observed value calculated for tensile strength of spruce wood.

\section{Conclusions}

Implementation of infrared techniques such as FT-NIR in to timber engineering and mechanical testing of wood provides very essential supplement to the typical information collected during standard tests. It extends the human perception into something "we can not see with naked eye". Investigations of wood changes (on the molecular level) by means of infrared spectroscopy provides new light in to better understanding the physics of the material behavior exposed to mechanical stresses. It was possible to predict stress level of wood during tension by applying proper chemometric analysis (partial least square (PLS) models). However, detailed interpretation of the constitutive components, and its interrelations, requires additional intensive research. Nevertheless, on the base of the research here presented, it seems that prediction of the mechanical stress on the basis of the infrared spectra to be possible. Practical implementation of FT-NIR spectroscopy in to timber engineering and mechanical testing of wood provides very essential supplement to the typical information collected traditionally during standard tests.

Acknowledgements: Part of this work has been conducted within the framework of project SWORFISH (team 2009 incoming (CALL 2) and Trentino-PCOFUND-GA-2008-226070) cofinanced by Provincia Autonoma di Trento. 


\section{References}

[1] G. Tampone, Mechanical Failures of the Timber Structural Systems From Material to Structure Mechanical Behaviour and Failures of the Timber Structures, ICOMOS IWC - XVI International Symposium, Italy (2007).

[2] N. Lombardini, Assessment of Deformability and Collapse Load of Timber Structures Built According to Treatises of the First Half of Nineteenth Century: the Case of the Truss Structures on the Roof of the Town Hall in Ravenna, ICOMOS IWC - XVI International Symposium, Italy (2007).

[3] M. Zborowska, A. Sandak, J. Sandak, S. Borysiak, W. Pradzynski, Characterization of archaeological wood degradation with selected non-destructive methods, proceedings of International Conference: Wooden Cultural Heritage: Evaluation of Deterioration and Management of Change, (2009).

[4] S. Tsuchikawa, A Review of Recent Near Infrared Research for Wood and Paper, Applied Spectroscopy Reviews, 42 (2007) 43-71.

[5] N. Gierlinger, M. Schwanninger, A. Reinecke, I. Burgert, Molecular changes during tensile deformation of single wood fibres followed by Raman microscopy, Biomacromolecules. 7 (2006) 2077-2081.

[6] J. Sandak, A. Sandak, M. Negri, Mechanical testing of wood assisted by infrared spectroscopy and thermal imaging. 11th World Conference on Timber Engineering, Italy (2010)

[7] N. Andre, N. Labbe, TG. Rials, S.S. Kelley, Assessment of wood load condition by Near Infrared (NIR) spectroscopy, J Mater Sci, 41,7 (2006) 1879-1886.

[8] M. Schwanninger, J.C. Rodrigues, K. Fackler, A review of band assignments in near infrared spectra of wood and wood components, J Near Infrared Spec, 19,5 (2011) 287-308. 